\title{
Prokaryotic Diversity of the Tucuruí Hydropower Plant Reservoir in the Brazilian Amazon
}

\author{
Rafael A. Baraúna \\ Universidade Federal do Pará, Instituto de Ciências Biológicas \\ Postal Code 479, Pará State, Brazil
}

Tel: 55-91-3201-8416_E-mail: r.a.barauna@gmail.com

Diego A Graças (Corresponding author)

Universidade Federal do Pará, Instituto de Ciências Biológicas

Postal Code 479, Pará State, Brazil

Tel: 55-91-3201-8416_E-mail: diego.a87@gmail.com

Paulo R. Miranda

Universidade Federal do Pará, Instituto de Ciências Biológicas

Postal Code 479, Pará State, Brazil

Tel: 55-91-3201-8416_E-mail: paulorobertom@gmail.com

Rubens Ghilardi Jr.

Centrais Elétricas do Norte do Brasil S.A - Eletronorte

Cep 70.716-901 - Brasília/DF

Tel: 55-91-3201-8416 E-mail: guilhardi@eln.gov.br

Maria Silvanira Barbosa

Laboratório de Polimorfismo de DNA

Universidade Federal do Pará, Instituto de Ciências Biológicas

Postal Code 479, Pará State, Brazil

Tel: 55-91-3201-8416Ｅ-mail: nirabarbosa@gmail.com 
Maria P. C. Schneider

\author{
Laboratório de Polimorfismo de DNA \\ Universidade Federal do Pará, Instituto de Ciências Biológicas \\ Postal Code 479, Pará State, Brazil
}

Tel: 55-91-3201-8416_E-mail: paula@ufpa.br

\begin{abstract}
Artur Silva
Laboratório de Polimorfismo de DNA

Universidade Federal do Pará, Instituto de Ciências Biológicas

Postal Code 479, Pará State, Brazil
\end{abstract}

Tel: 55-91-3201-8416 E-mail: asilva@ufpa.br

\begin{abstract}
Received: October 30, 2012 Accepted: December 12, 2012 Published: January 1, 2013
doi:10.5296/ast.v1i1.2981 URL: http://dx.doi.org/10.5296/ast.v1i1.2981
\end{abstract}

\begin{abstract}
To perform the a culture-independent characterization of the microbial diversity observed within a water body in the Amazon, we constructed a library of bacterial and archaeal $16 \mathrm{~S}$ rRNA genes isolated from environmental samples from the Tucuruí Hydroelectric Plant reservoir. The sampling locations included the upstream compartment (MR), which is located $50 \mathrm{~km}$ upstream of the dam, and Itupiranga (ITU), which is located upstream on the Tocantins River at the inlet of the reservoir. The bacterial phyla Cyanobacteria (46\%), Proteobacteria $(12 \%)$, Actinobacteria (9\%), Bacteroidetes (4\%) and Verrucomicrobia (2\%) were identified at the MR. Phyla including the Chloroflexi, Acidobacteria, Planctomycetes and TM6 candidate division were observed at a frequency of $<2 \%$. The phyla Cyanobacteria $(62 \%)$, Proteobacteria (5\%) and Actinobacteria (7\%) were identified at ITU. The archaeal diversity was limited to the Euryarchaeota and Crenarchaeota phyla; the latter was found in greater abundance and mainly consisted of uncultured phylotypes. In both regions, $25 \%$ and $95 \%$ of the bacteria and archaea, respectively, that were identified were previously unclassified. The phylum Cyanobacteria was almost exclusively represented by the genus Synechococcus sp.; however, a wide variety of microorganisms from the phylum Proteobacteria were identified from the classes alpha, gamma, delta and beta proteobacteria, with the latter being the most abundant. This study demonstrates that high prokaryotic diversity exists in the Amazonian rivers in addition to the previously reported megadiversity of fauna and flora.
\end{abstract}

Keywords: Amazon, Archaea, Bacteria, Hydropower reservoir, Metagenome, 16S rDNA 


\section{Introduction}

Despite the current knowledge of the megadiversity of animals and plants in the Amazon, little is known about the microbial diversity (Borneman \& Triplett, 1997; Fiore et al., 2005; Hungria et al., 2005; Fierer \& Jackson, 2006; Lima-Bittencourt, 2007).

The rivers that form the Amazon basin drain an area that is greater than $7 \times 106 \mathrm{~km} 2$ and discharge approximately $18 \%$ of all fresh water in the world into the oceans (Benner, 1995). These freshwater habitats are fundamentally important to humanity and are directly related to the maintenance of the Amazonian megadiversity and the density of rainfall on the continent; these rivers also provide an energy source through the use of hydroelectric power plants (Fearnside, 1989; Junk \& Mello, 1990; Fearnside, 1993).

The Brazilian energy matrix is $88 \%$ dependent on hydropower, and the Tucuruí Hydroelectric Power Station (HPS-Tucuruí) $\left(3^{\circ} 43^{\prime}\right.$ and $5^{\circ} 15^{\prime} \mathrm{S}$ and $49^{\circ} 12^{\prime}$ and $50^{\circ} 00^{\prime} \mathrm{W}$ ) is the primary genuinely national hydropower generator. In addition to the plants that are currently in operation, more than a dozen hydropower plant projects are under construction, and a similar number of new projects are planned for the Brazilian Amazon, which has the potential to provide $52 \%$ of the nation's hydropower. The construction of the HPS-Tucuruí resulted in the flooding of approximately $2,430 \mathrm{~km}^{2}$ of forest and the formation of a reservoir that has a total volume of 50,290 x $106 \mathrm{~m}^{3}$ and is $180 \mathrm{~km}$ in length (Junk \& Mello, 1990; Tundisi, 2007).

The construction of hydroelectric dams along the Amazon is responsible for many environmental changes both above and below the dams. Some of the impacts of these buildings are directly related to global climate change through the production of greenhouse gases. For example, the Tucuruí area that is now occupied by the reservoir was previously an ombrophilous forest. When this area was submerged, a large amount of organic matter was converted to $\mathrm{CO}_{2}$ and $\mathrm{CH}_{4}$ (Fearnside, 1995; Fearnside, 1997).

The analysis of microbial communities using cloned fragments of collective genomes has been the subject of intensive research in soil (Handelsman, 1998), marine (Venter et al., 2004), river (Gonzalez-Toril, 2003), wetland (Dedysh, 2006) and airborne (Tringe et al., 2008) ecosystems.

Estimates have been performed using DNA reassociation kinetics that predict that $99.9 \%$ of the pristine soil microbial diversity is unknown (Gans, 2005). Amann and colleagues (Amann et al., 1995) estimate that our knowledge of microbial diversity in mesotrophic lakes does not exceed $1 \%$ of the real diversity. Approaches based on molecular biology techniques have been demonstrated to be the most effective for determining the richness of diversity and the structure of microbial communities (Suzuki, 1998). To study this diversity in the natural environment, pioneering work using $16 \mathrm{~S}$ rDNA has resulted in a new system for the classification of life based on molecular sequences and structures that was proposed because these data are more revealing than approaches that are dependent on culturing (Woese, 1990). The present work contributes to the knowledge of microbial diversity through the sequencing of $16 \mathrm{~S}$ rDNA fragments found in ecogenomes from lakes in this region of the world. Thus, we chose to investigate the reservoir that was created by the HPS-Tucuruí using two locations: 


\section{Macrothink}

a site at the Tocantins river and another site in the middle of the lake.

\section{Research Methods}

\subsection{Location and Sample Collection}

The HPS-Tucuruí is located in the Eastern Brazilian Amazon where the river characteristics are suitable for power generation. This reservoir is located in the Araguaia basin on the lower course of the Tocantins River (Fig. 1).
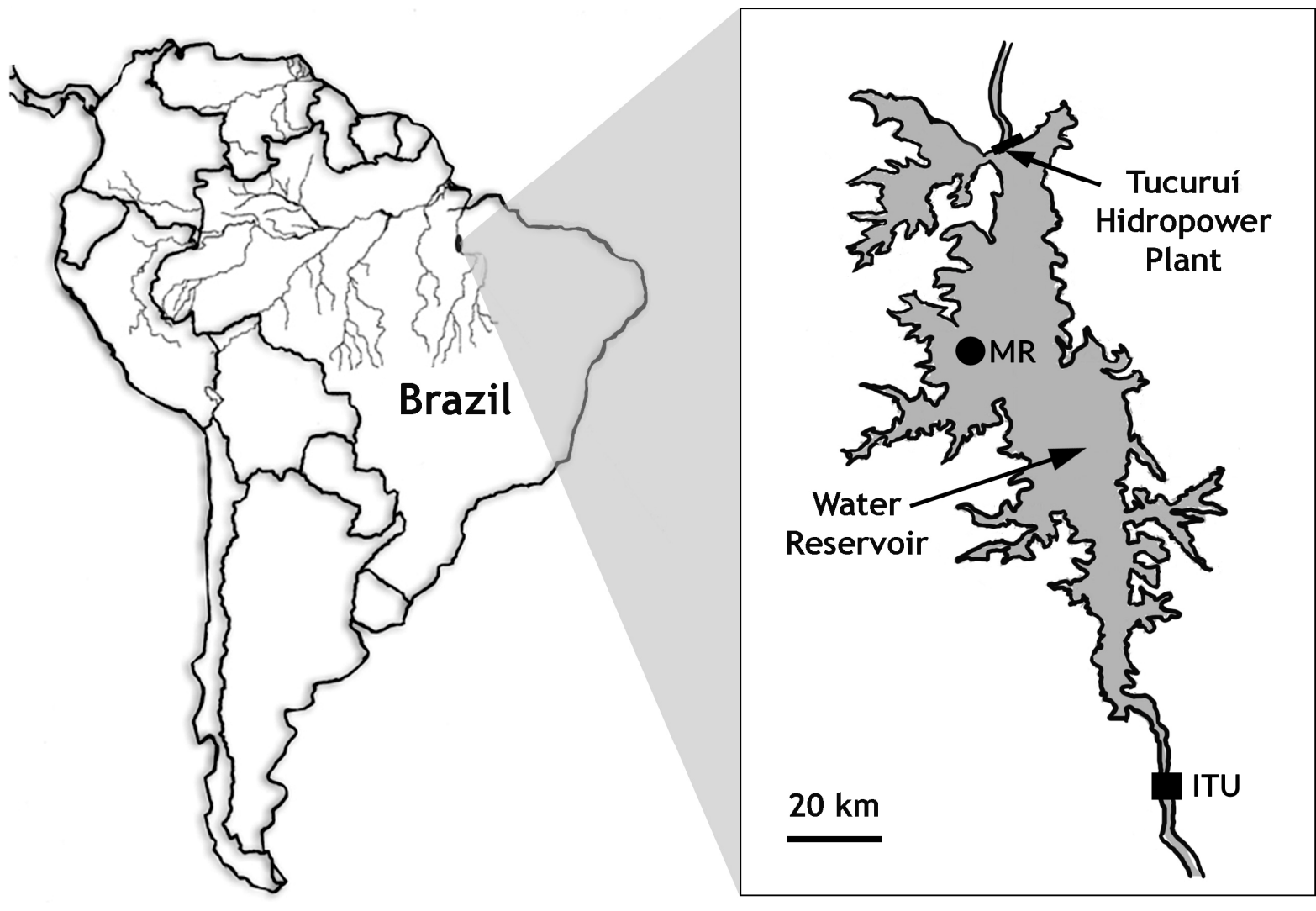

Figure 1. HPS Tucuruí geographical location

Description: Geographical location of the Tucuruí Hydroelectric Power Station on the Tocantins River in northern Brazil with its respective sampling points; the Upstream Compartment (MR $\odot$ ) and Itupiranga (ITU $\square)$ that were used in the metagenomic analysis.

To analyze the microbial communities and to determine the physicochemical characteristics of the reservoir, specific sampling regions established by the Limnology/Water Quality Program of the HPS-Tucuruí Centre of Environmental Protection (Centro de Proteção Ambiental - CPA) were used. The following sampling points were used in this study: the upstream compartment (MR) $\left(04^{\circ} 13^{\prime} 7^{\prime \prime} \mathrm{S}\right.$ and $\left.49^{\circ} 42^{\prime} 9.2^{\prime \prime} \mathrm{W}\right)$, which is located $50 \mathrm{~km}$ from the dam, and Itupiranga (ITU) $\left(05^{\circ} 08^{\prime} 0.2^{\prime}\right.$ S and $\left.49^{\circ} 19^{\prime} 14,1^{\prime \prime} \mathrm{W}\right)$, which is located upstream of the reservoir (Fig. 1). 
A total of $20 \mathrm{~L}$ of water was collected from each sampling site. The photic layer was sampled at ITU, and a pooled sample of the photic and aphotic layer was collected at MR $(0-20 \mathrm{~m})$; these samples were collected using a Van Dorn bottle and transferred to amber glass flasks.

At the collection site, the water temperature was measured, and sub-samples were taken for the analysis of $\mathrm{pH}$, optical density, ammonium, nitrate, phosphate and turbidity. The collection period in September 2006 corresponded to the Amazonian summer.

\subsection{DNA Extraction}

The separation of the microorganisms was performed using three parallel filtrations. In the first step we used a Büchner funnel with qualitative paper. The water was subsequently filtered under negative pressure through a sterile nitrocellulose membrane with a pore size of $0.8 \mu \mathrm{m}$ (Millipore, Brazil) and was filtered again under the same conditions using a $0.22 \mu \mathrm{m}$ membrane (Whatman, Germany). The membranes were perforated and transferred to a centrifuge tube containing $40 \mathrm{ml}$ of a DNA preservation solution $(50 \mathrm{mM}$ Tris $\mathrm{HCl}, 500 \mathrm{mM}$ $\mathrm{NaCl}, 125 \mathrm{mM}$ EDTA pH 8.0). The tubes were shaken overnight at room temperature on an orbital shaker. Precipitation of the cells was performed by centrifugation at $4000 \mathrm{rpm}$ for 20 minutes at room temperature.

Environmental DNA was isolated using the UltraClean ${ }^{\mathrm{TM}}$ Soil DNA Kit (MoBio, USA) according to the manufacturer's specifications. DNA quantification was performed using UV spectrometry.

\section{$2.316 S$ rRNA Gene Amplification Using the Polymerase Chain Reaction (PCR)}

PCR was performed to amplify 16S rRNA gene fragments. A PTC 200 thermocycler (MJ Research, UK) was used with the primers 8f (5'-AGAGTTTGATYMTGGCTCAG-3'), 1492r (5'-CGGTTACCTTGTTACGACTT-3') and S16.5-F3 (5'-GCCAGCCGCGGTAATAC-3') for bacteria and 341f (5'-CCTAYGGGGYGCASCAGGCG-3') and 1407r (5'-GACGGGGGTGWGTRCAA-3') for archaea. PCR started with a denaturation step at $94^{\circ} \mathrm{C}$ for $5 \mathrm{~min}$ followed by 30 cycles of $94^{\circ} \mathrm{C}$ for $1 \mathrm{~min}, 55^{\circ} \mathrm{C}$ for $1 \mathrm{~min}$ and $72^{\circ} \mathrm{C}$ for $90 \mathrm{~s}$ and a final extension of $10 \mathrm{~min}$ at $72^{\circ} \mathrm{C}$. The amplification solution had a final volume of 50 $\mu \mathrm{L}$ and contained the following reagents: $10 \times$ buffer $[22 \mathrm{mM}$ Tris- $\mathrm{HCl}(\mathrm{pH} 8.4), 55 \mathrm{mM}$ $\mathrm{KCl}$ ], $2.5 \mathrm{mM} \mathrm{MgCl} 2,0.2 \mathrm{mM}$ dNTPs, $5 \mu \mathrm{M}$ of each primer and $2 \mathrm{U}$ of Platinum Taq DNA polymerase (Invitrogen, USA).

The amplicons were visualized by electrophoresis on a $\%$ agarose gel (GE Healthcare, USA). Fragments in the range of approximately $1.5 \mathrm{~kb}$ for bacteria and $1 \mathrm{~kb}$ for archaea were excised from the gel and purified using the GFX PCR DNA and Gel Band Purification Kit (GE Healthcare, USA).

\subsection{Cloning and Sequencing}

The amplicons were ligated into the $\mathrm{pGEM}^{\circledR}-\mathrm{T}$ Easy Vector System (Promega Corp., USA) according to the manufacturer's instructions and were subsequently transformed into DH5 $\alpha$ Escherichia coli (Stratagene, USA) using an electroporator (GIBCO BRL, USA). The transformed bacteria were plated on semi-solid LB agar (DIFCO, USA) containing ampicillin 
(EMS, Brazil) and X-gal (GE Healthcare, USA). Recombinant clones were isolated by blue and white screening and grown in LB ampicillin liquid medium (DIFCO, USA) in deep well plates with shaking in an orbital incubator at $200 \mathrm{rpm}$ for 16 hours at $37^{\circ} \mathrm{C}$. The isolation of recombinant nucleic acids was performed using an alkaline lysis miniprep, and the vectors were purified using MAGV N22 membranes (Millipore, USA).

The sequencing reaction was performed using the DYEnamic ${ }^{\mathrm{TM}}$ ET Dye Terminator Kit according the manufacturer's instructions (GE Healthcare, USA) and the primers that were used for the PCR step. Sequencing electrophoresis was performed using a MegaBACE 1000 automatic nucleic acid detection system according to the manufacturer's instructions (GE Healthcare, USA).

\subsection{Data Analysis}

For the bacterial sequences, the Sequencher 4.0.5 (Gene Codes Corp.) and BioEdit (www.mbio.ncsu.edu/BioEdit/bioedit.html) programs were used for the assembly and manual editing of contigs of approximately $1200 \mathrm{bp}$, and Mallard software (Ashelford, 2006) was used to detect chimeric sequences and possible sequencing and editing anomalies. The sequences were aligned to sequences from the SILVA database using the Sina WebAligner online tool (Pruesse et al., 2007). From this alignment, distance matrices were calculated and corrected using the Jukes-Cantor algorithm in the DNAdist program of the Phylip package (http://evolution.genetics.washington.edu/phylip.html). These files were used as input for the DOTUR program version 1.53 (Schloss \& Handelsman, 2005), which was used to calculate the diversity indices of the samples using the Furthest Neighbor method.

The estimated number of operational taxonomic units (OTUs) was calculated using the nonparametric estimators ACE and Chao1. The Good's C (Good, 1953) and Chao et al. $\mathrm{C}_{\mathrm{ACE}}$ (Chao, 1993) nonparametric coverage estimators were used. Sequence classification was performed using the SINA WebAligner tool, which aligned the experimental sequences with the most closely related sequences in the SILVA 16S rRNA database. Archeal domain sequences were submitted to the MyRDP pipeline (Cole et al., 2007) using PHRED software for quality analysis; only those sequences with a score equivalent to 20 were used. The LUCY software was used to remove sequences derived from the pGEM-T Easy vector.

Dendrograms were constructed using the neighbor-joining method and were generated in the Phylip package using the Jukes-Cantor model to calculate the distance between the sequences. A bootstrap analysis of the arrangements was performed with 1000 replicates, and consensus values greater than $50 \%$ were shown at the branches of the cladograms, which were viewed using the Figtree application (http://tree.bio.ed.ac.uk/). The sequences obtained for this study were submitted to GenBank with the accession numbers EU592502 to EU592963

\section{Results}

The physicochemical parameters for both sampling points are described in Table 1. The low concentration of nitrates at the water surface suggests that ammonium is the primary nitrogen source used by the microorganisms in this layer (Table 1). 
Table 1. Physicochemical parameters

\begin{tabular}{lccccccc}
\hline & $\mathrm{pH}$ & $\mathrm{OD}(\mathrm{mg} / \mathrm{mL})$ & $\mathrm{NH}_{4}{ }^{+}(\mu \mathrm{g} / \mathrm{L})$ & $\mathrm{NO}_{3}{ }^{-}(\mu \mathrm{g} / \mathrm{L})$ & $\mathrm{PO}_{4}{ }^{-}(\mu \mathrm{g} / \mathrm{L})$ & Turbidez $(\mathrm{NTU})$ & Temperatura $\left({ }^{\circ} \mathrm{C}\right)$ \\
\hline MR 0.00m & 7.37 & 5.96 & 8.48 & $0.0^{*}$ & 13.05 & 11.76 & 32.60 \\
MR 26.0m & 6.69 & 0.00 & 1402.21 & 35.89 & 72.97 & 25.30 & 29.20 \\
ITU 0.00m & 7.54 & 6.99 & 10.70 & $0.0^{*}$ & 13.05 & 22.70 & 29.80 \\
\hline
\end{tabular}

Description: The measurements were done by the Centre of Environmental Protection as part of the Limnology/Water quality control. *Values below detection level.

The metagenomic library had a total of 345 bacterial clones of approximately $1200 \mathrm{bp}$; 61 of these clones corresponded to ITU, and 284 corresponded to MR. In addition, a total of 117 partial archaeal sequences of approximately $400 \mathrm{bp}$ were obtained: 37 were collected from ITU, and 80 were collected from MR. The taxonomic relationships demonstrate that the OTUs were affiliated with nine bacterial phyla and two archaeal phyla (Figs. 2 and 3).

In the two sampled areas, approximately $25 \%$ of the bacteria and $95 \%$ of the archaea were identified as unclassified environmental taxa. The bacterial phylum with the highest relative abundance at both sampling points was the Cyanobacteria. However, a considerable abundance of Actinobacteria and Proteobacteria was also observed. Additionally, Verrucomicrobia and Bacteroidetes were observed only in the MR. The phyla Chloroflexi, Acidobacteria, Planctomycetes and the TM6 candidate division were detected in the metagenome of the MR sampling point. During the characterization of the archaeal phylotypes, representatives from the phyla Euryarchaeota and those corresponding to uncultured archaea were identified from both sampling points (Table 2).

Table 2. Accession number of each unique OTU with their closest relative organism from ARB-SILVA

\begin{tabular}{|c|c|c|c|c|c|}
\hline $\begin{array}{l}\text { GenBank } \\
\text { Accession }\end{array}$ & $\begin{array}{l}\text { No. of } \\
\text { Clones }\end{array}$ & $\begin{array}{l}\text { Maximum } \\
\text { Identity } \\
(\%)\end{array}$ & $\begin{array}{l}\text { Closest Relative Organism } \\
\text { from ARB-Silva }\end{array}$ & $\begin{array}{l}\text { Accession } \\
\text { NCBI \& } \\
\text { CAMERA }\end{array}$ & $\begin{array}{l}\text { Environmental Location of the Closest } \\
\text { Relative Organism }\end{array}$ \\
\hline EU592838 & 1 & 96 & $\begin{array}{l}\text { uncultured } \\
\text { bacterium }\end{array}$ & AM292624 & Uranium mining waste pile, Germany (unpublished) \\
\hline EU592601 & 5 & 88 & Streptomyces flavidofuscus & AY999914 & Data not found \\
\hline EU592628 & 4 & 98 & uncultured actinobacterium & AJ575501 & Rimov reservoir water, Czech Republic (Warnecke, 2004) \\
\hline EU592635 & 3 & 99 & uncultured actinobacterium & EU117783 & Little Arbor Vitae Lake, Wisconsin, USA (Newton, 2007) \\
\hline EU592590 & 3 & 97 & Mycobacteriaceae bacterium & DQ490438 & Kilauea volcano deposits, Hawaii, USA (unpublished) \\
\hline EU592623 & 3 & 95 & uncultured actinobacterium & EU117723 & Hook Lake water, Wisconsin, USA (Newton, 2007) \\
\hline EU592596 & 2 & 97 & uncultured actinobacterium & EF520353 & $\begin{array}{l}\text { Acid-impacted Adirondack Lake, New York, USA (Percent, } \\
\text { 2008) }\end{array}$ \\
\hline EU592593 & 2 & 88 & Streptomyces flavidofuscus & AY999914 & Data not found \\
\hline EU592508 & 1 & 99 & uncultured actinobacterium & EU117871 & Mirror Lake water, Wisconsin, USA (Newton, 2007) \\
\hline EU592625 & 1 & 98 & uncultured actinobacterium & AJ575506 & Lake Schoehsee water, Germany (Wercecke, 2004) \\
\hline EU592597 & 1 & 97 & uncultured actinobacterium & AY948059 & Parker River, Massachussetts, USA (Crump, 2005) \\
\hline EU592504 & 1 & 97 & uncultured actinobacterium & AJ575506 & Lake Schoehsee water, Germany (Wercecke, 2004) \\
\hline EU592634 & 1 & 96 & uncultured actinobacterium & EU117954 & Trout Lake water, Wisconsin, USA (Newton, 2007) \\
\hline EU592502 & 1 & 95 & uncultured actinobacterium & EU117730 & Ike Walton Lake water, Wisconsin, USA (Newton, 2007) \\
\hline EU592511 & 1 & 95 & uncultured actinobacterium & EU117897 & Red Cedar Lake water, Wisconsin, USA (Newton, 2007) \\
\hline EU592624 & 1 & 92 & uncultured actinobacterium & AJ575506 & Lake Schoehsee water, Germany (Wercecke, 2004) \\
\hline EU592563 & 5 & 97 & Bacteroidetes & DQ463716 & Lake Tanganyika oxic epilimnion, Tanzania (unpublished) \\
\hline
\end{tabular}




\begin{tabular}{|c|c|c|c|c|c|}
\hline EU592570 & 4 & 98 & $\begin{array}{l}\text { bacterium } \\
\text { uncultured } \\
\text { bacterium }\end{array}$ & DQ463729 & Lake Tanganyika oxic epilimnion, Tanzania (unpublished) \\
\hline EU592569 & 1 & 98 & Flavobacteria bacterium KF030 & AB269814 & Lake Kasumigaura water, Japan (Watanabe, 2009) \\
\hline EU592568 & 1 & 93 & $\begin{array}{l}\text { uncultured } \\
\text { bacterium }\end{array}$ & AY947919 & Ipswich River, Massachussetts, USA (Crump, 2005) \\
\hline EU592821 & 1 & $\mathrm{n} / \mathrm{a}$ & $\begin{array}{l}\text { uncultured candidate division TM6 } \\
\text { bacterium }\end{array}$ & DQ676372 & Suboxic freshwater-pond, France (Briée, 2007) \\
\hline EU592667 & 1 & 91 & uncultured Chloroflexi bacterium & DQ501314 & Lake Grosse Fuchskuhle water, Germany (unpublished) \\
\hline EU592712 & 83 & 99 & Synechococcus sp. MH305 & AY224198 & Central European Subalpine Lakes (Crosbie, 2003) \\
\hline EU592688 & 35 & 98 & Synechococcus sp. 0BB26S03 & AJ639899 & Bubano Basin, Italy (unpublished) \\
\hline EU592523 & 20 & 99 & Synechococcus sp. LBG2 & AF330249 & Brackish Baltic Sea (Ernst, 2003) \\
\hline EU592708 & 10 & 97 & Synechococcus sp. MW28B3 & AY151235 & Central European Subalpine Lakes (Crosbie, 2003) \\
\hline EU592527 & 9 & 97 & Synechococcus sp. 0BB26S03 & AJ639899 & Bubano Basin, Italy (unpublished) \\
\hline EU592522 & 2 & 97 & Synechococcus sp. MW25B5 & AY151233 & Central European Subalpine Lakes (Crosbie, 2003) \\
\hline EU592672 & 2 & 97 & Synechococcus sp. MH305 & AY224198 & Central European Subalpine Lakes (Crosbie, 2003) \\
\hline EU592546 & 1 & 98 & Synechococcus sp. PS721 & AF216954 & Lake Biwa water, Shiga, Japan (Robertson, 2001) \\
\hline EU592537 & 1 & 98 & uncultured bacterium & EU804045 & Lake Gatun water, Panama (Shaw, 2008) \\
\hline EU592532 & 1 & 97 & Merismopedia sp. CENA106 & EF088332 & Waste satabilization pond, Brazil (unpublished) \\
\hline EU592558 & 1 & 95 & Synechococcus sp. MH305 & AY224198 & Central European Subalpine Lakes (Crosbie, 2003) \\
\hline EU592536 & 1 & 97 & uncultured bacterium & EU804045 & Lake Gatun water, Panama (Shaw, 2008) \\
\hline EU592555 & 1 & 95 & Synechococcus sp. PS721 & AF216954 & Lake Biwa water, Shiga, Japan (Robertson, 2001) \\
\hline EU592543 & 1 & 96 & uncultured bacterium & EU803469 & Lake Gatun water, Panama (Shaw, 2008) \\
\hline EU592823 & 1 & 88 & uncultured bacterium & EU803469 & Lake Gatun water, Panama (Shaw, 2008) \\
\hline EU592606 & 15 & 99 & uncultured bacterium & DQ520197 & Hypertrophic Meiliang Bay, China (Wu, 2007) \\
\hline EU592503 & 8 & 99 & uncultured bacterium & $\mathrm{AB} 154300$ & Lake Kasumigaura water, Japan (unpublished) \\
\hline \multirow[t]{2}{*}{ EU592636 } & 6 & 99 & marine metagenome & AACY0201 & Marine metagenome (Venter, 2004) \\
\hline & & & & 73194 & \\
\hline EU592680 & 4 & 96 & uncultured bacterium & DQ444431 & Lake Tanganyika, Tanzania (Schubert, 2006) \\
\hline EU592824 & 4 & 84 & uncultured soil bacterium & DQ298008 & Hydrocarbon Contaminated Soil, USA (unpublished) \\
\hline EU592676 & 3 & 98 & uncultured bacterium & DQ444431 & Lake Tanganyika, Tanzania (Schubert, 2006) \\
\hline EU592598 & 3 & 96 & bacterium $\mathrm{rJ} 7$ & AB021325 & Phenol-digesting activated Sludge, Japan (Watanabe, 1999) \\
\hline EU592673 & 3 & 94 & uncultured bacterium & AJ538354 & Lake Cadagno, Switzerland (unpublished) \\
\hline EU592685 & 3 & 88 & uncultured bacterium & DQ906072 & Tinto River Rhizosphere, Spain (unpublished) \\
\hline \multirow[t]{2}{*}{ EU592828 } & 2 & 100 & marine metagenome & AACY0201 & Marine metagenome (Venter, 2004) \\
\hline & & & & 79327 & \\
\hline \multirow[t]{2}{*}{ EU592646 } & 2 & 98 & marine metagenome & AACY0234 & Marine metagenome (Venter, 2004) \\
\hline & & & & 37746 & \\
\hline EU592576 & 2 & 97 & uncultured bacterium & EU133614 & Kessler Farm soil, Oklahoma, USA (Elshahed, 2008) \\
\hline EU592831 & 2 & 93 & uncultured Crater Lake bacterium & AF316766 & Ultra-oligotrophic Crater Lake, Oregon, USA (Urbach, 2001) \\
\hline EU592665 & 2 & 91 & bacterium $005-\mathrm{D}$ & AY661916 & Groundwater contamined, USA (Fields, 2005) \\
\hline EU592643 & 1 & 99 & uncultured bacterium & EF157202 & Asphalts from Rancho La Brea Tar Pits, USA (Kim, 2007) \\
\hline \multirow[t]{2}{*}{ EU592818 } & 1 & 99 & marine metagenome & AACY0201 & Marine metagenome (Venter, 2004) \\
\hline & & & & 79327 & \\
\hline \multirow[t]{2}{*}{ EU592833 } & 1 & 99 & marine metagenome & AACY0201 & Marine metagenome (Venter, 2004) \\
\hline & & & & 74722 & \\
\hline EU592594 & 1 & 98 & uncultured bacterium & AJ867924 & Alpine Lake Joeri XIII, Switzerland (unpublished) \\
\hline EU592510 & 1 & 98 & uncultured bacterium & DQ520196 & Hypertrophic Meiliang Bay, China (Wu, 2007) \\
\hline \multirow[t]{2}{*}{ EU592612 } & 1 & 97 & marine metagenome & AACY0201 & Marine metagenome (Venter, 2004) \\
\hline & & & & 81515 & \\
\hline EU592632 & 1 & 97 & uncultured bacterium & DQ520196 & Hypertrophic Meiliang Bay, China (Wu, 2007) \\
\hline EU592845 & 1 & 97 & uncultured bacterium & $\mathrm{AB} 154317$ & Lake Kasumigaura water, Japan (unpublished) \\
\hline EU592519 & 1 & 97 & uncultured bacterium & EF203205 & Lake Kastoria Sediment, Greece (unpublished) \\
\hline EU592587 & 1 & 96 & unidentified bacterium & AY345540 & Lake Wai'ele'ele water, Hawaii, USA (unpublished) \\
\hline EU592588 & 1 & 96 & uncultured bacterium & EF590055 & Songhua River sediment contamined with nitrobenzene $(\mathrm{Li}, 2008)$ \\
\hline EU592621 & 1 & 96 & uncultured bacterium & DQ520197 & Hypertrophic Meiliang Bay water, China (Wu, 2007) \\
\hline
\end{tabular}




\begin{tabular}{|c|c|c|c|c|c|}
\hline EU592817 & 1 & 96 & uncultured bacterium & EF157231 & Asphalts from Rancho La Brea Tar Pits, USA (Kim, 2007) \\
\hline EU592842 & 1 & 96 & uncultured bacterium & AY963365 & Humus and mineral soils forest, China (Chan, 2006) \\
\hline EU592514 & 1 & 96 & uncultured bacterium & DQ520165 & Hypertrophic Meiliang Bay water, China (Wu, 2007) \\
\hline EU592651 & 1 & 95 & uncultured bacterium & AB294330 & Deep coal seam groundwater, Japan (Shimizu, 2007) \\
\hline EU592586 & 1 & 94 & uncultured bacterium & AY328588 & Drinking water distribuition system, Ohio, USA (Williams, 2004) \\
\hline EU592653 & 1 & 94 & uncultured bacterium & AY706436 & TCE-contaminated groundwater, Ohio, USA (Humphries, 2005) \\
\hline EU592830 & 1 & 92 & uncultured Crater Lake bacterium & AF316767 & Ultra-oligotrophic Crater Lake, Oregon, USA (Urbach, 2001) \\
\hline EU592668 & 1 & 91 & uncultured bacterium & EU134543 & Kessler Farm soil, Oklahoma, USA (Elshahed, 2008) \\
\hline \multirow[t]{2}{*}{ EU592516 } & 1 & 91 & marine metagenome & AACY0201 & Marine metagenome (Venter, 2004) \\
\hline & & & & 74722 & \\
\hline EU592518 & 1 & 91 & uncultured bacterium & DQ520177 & Hypertrophic Meiliang Bay water, China (Wu, 2007) \\
\hline EU592820 & 1 & 90 & uncultured bacterium & EU134543 & Kessler Farm soil, Oklahoma, USA (Elshahed, 2008) \\
\hline \multirow[t]{2}{*}{ EU592517 } & 1 & 90 & marine metagenome & AACY0240 & Marine metagenome (Venter, 2004) \\
\hline & & & & 99985 & \\
\hline EU592520 & 1 & 90 & uncultured bacterium & DQ520197 & Hypertrophic Meiliang Bay water, China (Wu, 2007) \\
\hline EU592562 & 1 & 89 & uncultured bacterium & AY212691 & Water 10m downstream of manure (Simpson, 2004) \\
\hline EU592819 & 1 & 88 & uncultured bacterium & DQ413113 & Data not found \\
\hline EU592677 & 1 & 96 & uncultured planctomycete & AJ616263 & Elbe River biofilm, Germany (Bruemmer, 2004) \\
\hline EU592678 & 1 & 96 & uncultured planctomycete & AJ616263 & Elbe River biofilm, Germany (Bruemmer, 2004) \\
\hline EU592577 & 6 & 99 & uncultured alpha proteobacterium & DQ501352 & Lake Stechlin water, Germany (unpublished) \\
\hline EU592659 & 5 & 99 & uncultured beta proteobacterium & AM849452 & Oligo-mesotrophic lake Piburger See, Austria (Salcher, 2008) \\
\hline \multirow[t]{2}{*}{ EU592654 } & 4 & 98 & proteobacterium & AJ876403 & Data not found \\
\hline & & & MWH-IPGL7W22 & & \\
\hline EU592645 & 4 & 99 & uncultured beta proteobacterium & AM849432 & Oligo-mesotrophic lake Piburger See, Austria (Salcher, 2008) \\
\hline EU592644 & 2 & 95 & Sphaerotilus sp. L19 & AB087568 & Data not found \\
\hline EU592584 & 2 & 99 & uncultured proteobacterium & DQ450165 & Shallow atlantic coastal lagoon (Piccini, 2006) \\
\hline EU592839 & 2 & 97 & $\begin{array}{l}\text { uncultured } \\
\text { proteobacterium }\end{array}$ & AY509440 & $\begin{array}{l}\text { Freshwater bacterioplankton of eutrophic lakes, Sweden (Eiler, } \\
\text { 2004) }\end{array}$ \\
\hline EU592574 & 1 & 95 & uncultured alpha proteobacterium & AJ518772 & Uranium mining waste pile, Germany (unpublished) \\
\hline EU592583 & 1 & 99 & uncultured alpha proteobacterium & AY948064 & Parker River, Massachussetts, USA (Crump, 2005) \\
\hline EU592589 & 1 & 98 & uncultured alpha proteobacterium & AY622245 & Surface soil of Oak Ridge Reservation, USA (Reardon, 2004) \\
\hline EU592560 & 1 & 97 & uncultured alpha proteobacterium & AY948064 & Parker River, Massachussetts, USA (Crump, 2005) \\
\hline EU592561 & 1 & 99 & uncultured alpha proteobacterium & DQ501352 & Lake Stechlin water, Germany (unpublished) \\
\hline \multirow[t]{2}{*}{ EU592521 } & 1 & 99 & proteobacterium & AJ876403 & Data not found \\
\hline & & & MWH-IPGL7W22 & & \\
\hline EU592652 & 1 & 93 & uncultured beta proteobacterium & AF529336 & Data not found \\
\hline EU592658 & 1 & 88 & Aquitalea magnusonii & DQ018117 & Humic-lake water sample, Wisconsin, USA (Lau, 2006) \\
\hline EU592669 & 1 & 98 & uncultured delta proteobacterium & EF520534 & $\begin{array}{l}\text { Acid-impacted Adirondack Lake, New York, USA (Percent, } \\
\text { 2008) }\end{array}$ \\
\hline EU592841 & 1 & 86 & uncultured delta proteobacterium & EU050845 & Sediment from the Kings Bay, Svalbard, Arctic (unpublished) \\
\hline EU592822 & 1 & $\mathrm{n} / \mathrm{a}$ & uncultured proteobacterium & $\mathrm{AF} 420354$ & $\begin{array}{l}\text { Guaymas Basin hydrothermally active sediments, Mexico (Teske, } \\
\text { 2002) }\end{array}$ \\
\hline EU592670 & 1 & 99 & Acinetobacter sp. DQ124 & DQ300355 & Data not found \\
\hline EU592671 & 1 & 96 & Acinetobacter sp. IGCAR-9/07 & EF517956 & Biofilm formed on modified titanium surface, India (unpublished) \\
\hline EU592835 & 4 & 95 & $\begin{array}{l}\text { uncultured } \\
\text { bacterium }\end{array}$ & EF520638 & $\begin{array}{l}\text { Acid-impacted Adirondack Lake, New York, USA (Percent, } \\
\text { 2008) }\end{array}$ \\
\hline EU592837 & 1 & 95 & unidentified eubacterium LD29 & AF009975 & Lake Loosdrecht water, Netherlands (Zwart, 1998) \\
\hline EU592846 & 1 & 94 & $\begin{array}{l}\text { uncultured } \\
\text { bacterium }\end{array}$ & AY509518 & $\begin{array}{l}\text { Freshwater bacterioplankton of eutrophic lakes, Sweden (Eiler, } \\
2004 \text { ) }\end{array}$ \\
\hline EU592947 & 133 & 97 & marine metagenome & JCVI_NT_1 & Lake Gatun water, Panama (unpublished) \\
\hline & & & & 1050101565 & \\
\hline & & & & 67 & \\
\hline EU592916 & 7 & 90 & uncultured euryarchaeote & AB243796 & Rice paddy soil, Japan (Sakai, 2007) \\
\hline
\end{tabular}

Description: Classification of unique OTUs obtained in the Tucuruí ecogenome that grouped with $3 \%$ dissimilarity compared with sequences deposited in the SILVA and CAMERA databases. 


\section{Macrothink

The statistical calculation of the diversity index was only performed for the bacterial domain because the archaeal diversity consisted of only two phylotypes. In the rarefaction curves, 26 species, 22 genera and eight phyla were identified in the sample collected from ITU (Fig. 4, A), and 77 species, 63 genera and 29 phyla were identified in the sample collected from MR (Fig. 4, B).

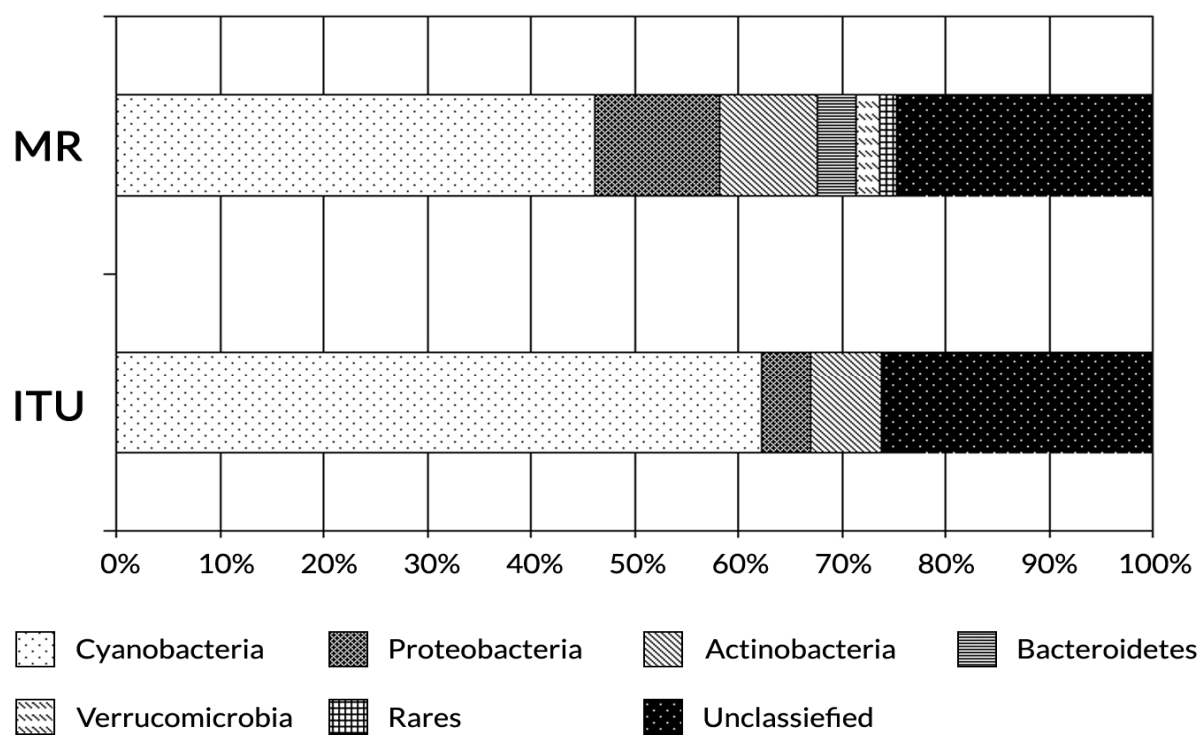

Figure 2. The bacterial diversity at phylum level found at MR and ITU points

Description: Diversity of bacterial phyla described from the different sampling points of the reservoir. *Among the rare phyla $(<2 \%)$ are Chloroflexi, Acidobacteria, Planctomycetes and a candidate for the TM6 division.

In both libraries, at a distance of 3\% between the sequences, the two curves were weakly curvilinear, which suggests that more exhaustive sampling would detect even more phylotypes at the two locations. The curves with $20 \%$ dissimilarity reached saturation, indicating that few additional phyla would be found with a greater sampling effort.

The coverages calculated using the Good's $\mathrm{C}$ estimator were $64 \%$ and $85 \%$ for ITU and MR, respectively. Lower values were obtained using the Chao $\mathrm{C}_{\mathrm{ACE}}$ estimator at $45 \%$ for ITU and $72 \%$ for MR. For dissimilarities of $3 \%$ and $20 \%$ between the sequences, the ACE richness estimator detected 210 and 14 OTUs, respectively, in ITU, and 145 and 39 OTUs, respectively, in MR. For dissimilarities of $3 \%$ and $20 \%$, the Chaol richness estimator predicted 142 and 10 OTUs in ITU and 152 and 40 OTUs, respectively, in MR (Fig. $04 \mathrm{C}$ and D). When the OTUs were grouped at 3\% dissimilarity, as defined using the DOTUR program, a total of 105 unique OTUs were identified; these OTUs were classified and are described in Table 2. 


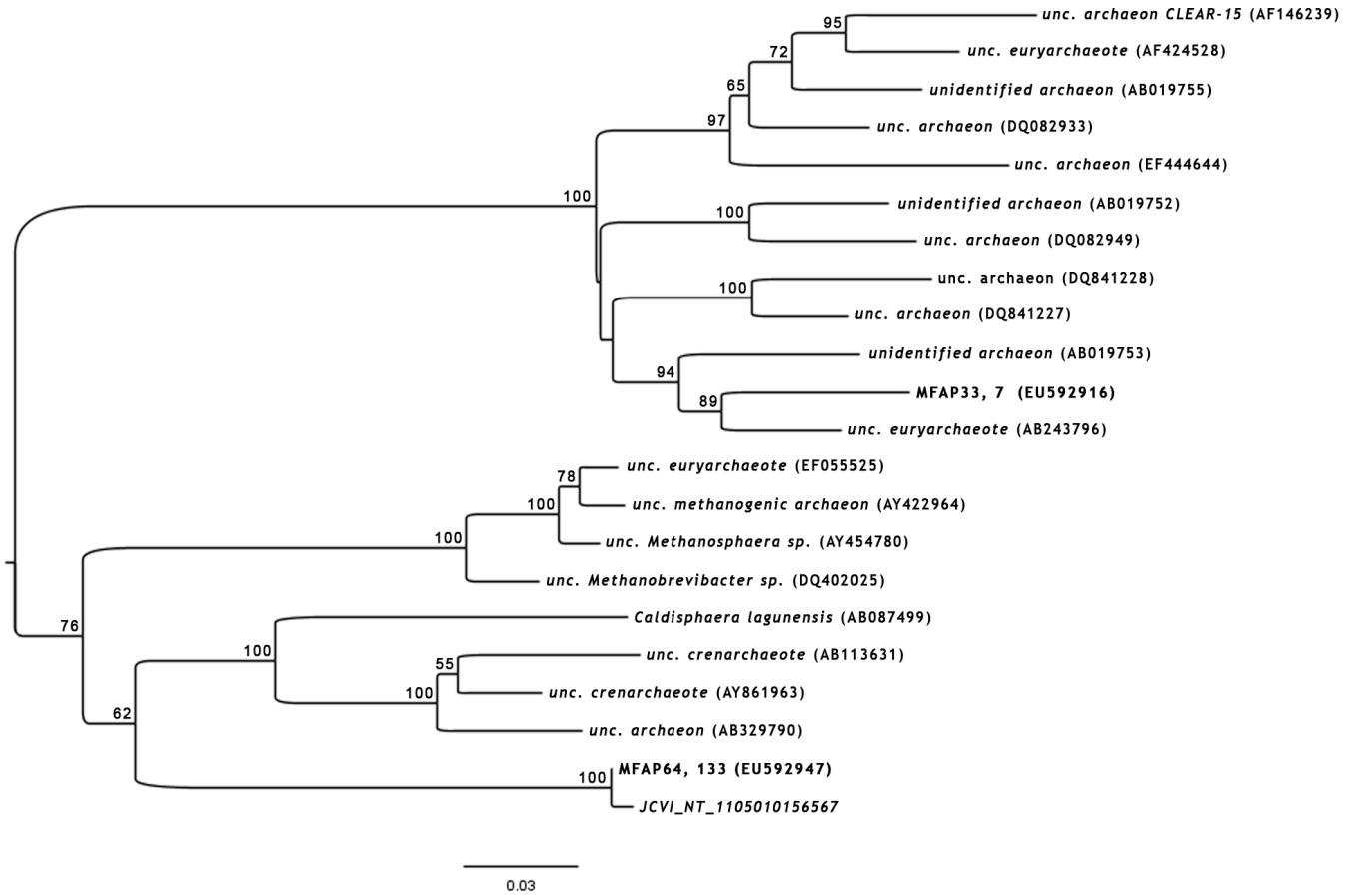

Figure 3. Phylogenetic analysis of Archaea sequences

Description: Archaeal phylogenetic tree showing the diversity found at the sampling points. Sequences that start with MFAP were obtained from the Tucurui metagenome. After the comma is the number of OTUs with 3\% dissimilarity and the GenBank accession number is in brackets.

(A)

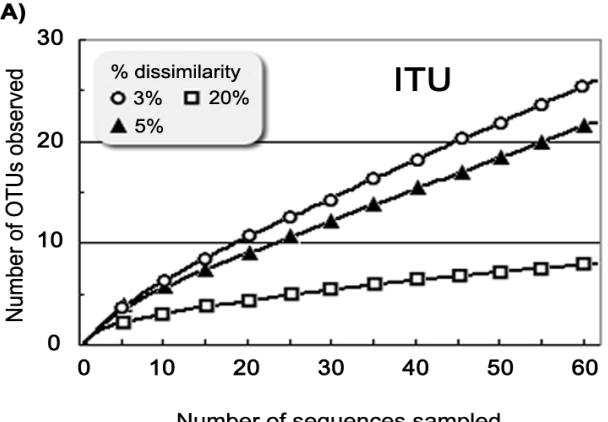

(C)

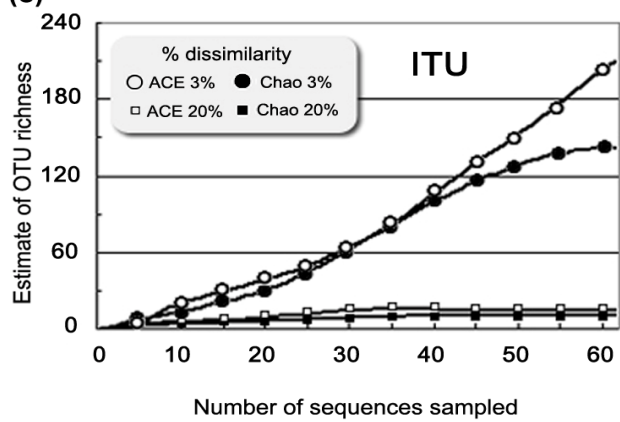

(B)

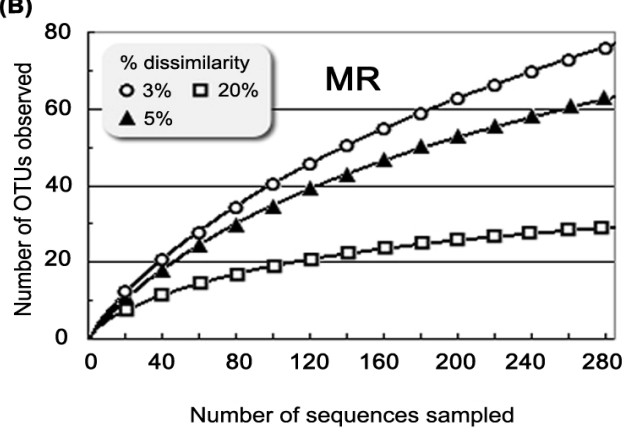

(D)

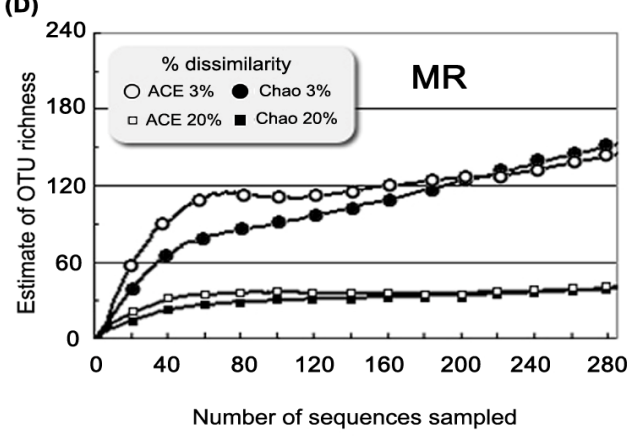

Figure 4. Rarefaction curves and estimators analysis

Description: Rarefaction curves and Ace and Chaol richness indices for the sampling points ITU and MR. The OTUs with $3 \%$ dissimilarity were classified at the species level, those with $5 \%$ dissimilarity were classified at the genus level and those with $20 \%$ dissimilarity were characterized at the phylum level. 


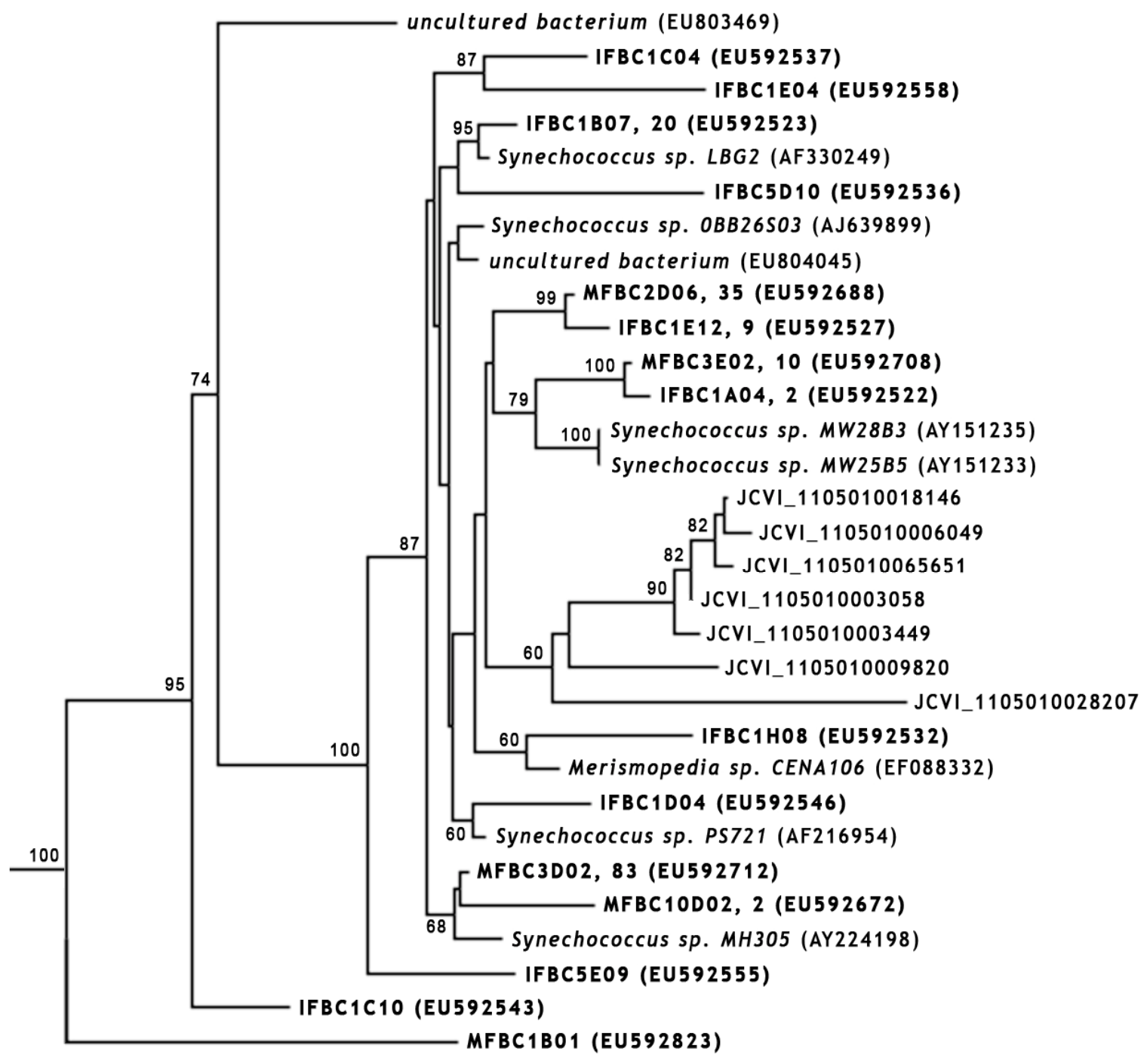

0.02

Figure 5. Phylogenetic analysis of Cyanobacteria sequences

Description: Phylogenetic tree of cyanobacteria with the 15 unique OTUs grouped with the closest sequences obtained from the SILVA and CAMERA databases. Sequences that start with MFBC or IFBC are from the Tucuruí metagenome and sequences that start with JCVI were obtained from CAMERA. Next to the name of the clones is the number of sequences that grouped with 3\% dissimilarity and in brackets is the GenBank accession number.

\section{Discussion}

Aquatic environments are less diverse relative to other environments such as soil or sediment and typically have an estimated phylotype richness of less than 200 (Curtis, 2002; Kemp \& 
Aller, 2004; Nold \& Zwart, 1998; Torsivik, 2002). Tucuruí Lake had a diversity of 142 to 210 OTUs at $3 \%$ dissimilarity. These values are within the expected range according to Kemp and Aller (2004). These diversity values could increase if the number of chimeras was fewer given that they comprised $7 \%$ and $12 \%$ of the total contigs obtained from ITU and MR, respectively.

In silico analysis revealed an abundance of bacterial species that corresponded to uncultured microorganisms or that were distant from sequences deposited in the 16S rRNA database. The low diversity of archaea identified in this study is probably due to the low number of sequences deposited in the database, especially with regard to those originating from equatorial areas of the ecosphere. The primary phyla that were characterized in the Tucuruí Lake ecogenome are discussed below.

\subsection{Cyanobacteria}

Cyanobacteria was the most predominant phylum at both sampling points. Despite its importance in the food chain, little is known about this group in the Brazilian Amazon (Fiore et al., 2005). The cyanobacteria observed in Tucuruí Lake corresponded almost entirely to the genus Synechococcus. This group and the genus Prochlorococcus are the dominant type of photoautotrophic picoplankton and are major contributors to global primary production (Waterbury, 1986). Nitrogen is one of the most abundant elements in Synechococcus cells and accounts for approximately 5-10\% of their dry cell weight (Bryant, 1994). The high ammonia concentration and the low frequency of other denitrifying groups may be primarily responsible for the targeted selection of this group. Other determinants for the successful colonization by this group are probably related to the sampling period, which is characterized by high luminosity and temperature (Table 1).

In our analysis, 164 of the 169 sequences that were classified as cyanobacteria belonged to the genus Synechococcus. At the MR region, the representatives of this genus are closely related to the Synechococcus species described in subalpine lakes of central European (Crosbie, 2003). Although Ernst (2003) suggested that these strains belonged to groups of cyanobacteria inhabiting mainly freshwater and saline lakes, this is contrary to the idea that many species of free-living microorganisms have a global ecological distribution (Finlay, 2002). The marine cyanobacteria described in the analysis by Venter et al. (2004) form clades distinct from those found in freshwater lakes including those described in the Tucuruí reservoir, as observed in Figure 05, reinforcing the concept that these species are distinct from the groups found in different environments. Other cyanobacteria that are part of the lake microbial community form distinct clades with high bootstrap values, suggesting a high diversity of phylotypes (Figure 05).

\subsection{Actinobacteria}

In our study, 15 actinobacteria phylotypes were detected at the two locations. The sequences that are most similar to those within the SILVA database are predominantly from freshwater lakes and belong to uncultivated lineages. The phylum actinobacteria is relatively diverse and contains several subgroups. Furthermore, little is known about their role in the environment. 
Several lineages have been defined as acI, acII, acIII and acIV, these clusters are clearly distinct from the lineages found in soil and marine habitats (Warnecke, 2004).

A total of 18 clones that are classified as uncultured bacteria, as represented by the accession numbers EU592606, EU592621, EU592514 and EU592520 (Table 02), are related to the Actinobacteria described in a study that was performed in Lake Taihu in China (Wu, 2007), in which the characterization of the ML-51.2-5 lineage within the Actinobacteria phylum was performed by phylogenetic analysis.

\subsection{Proteobacteria}

Among the most diverse and well-represented bacterial phylum in the databases is the Proteobacteria. This phylum has more than 460 genera and greater than 1600 species that were known as of 2002, which accounts for $40 \%$ of the publications regarding prokaryotic microorganisms (Kersters, 2006) and includes 44\% of the bacterial genome sequencing projects in the world (Liolios, 2007). In our analysis, representatives of the alpha- and betaproteobacteria classes were detected in the samples from both ITU and MR. Gamma and delta proteobacteria were also described in MR (Fig. 05). The presence of these classes at MR compared to ITU, which is a region with a less-modified landscape, may be related to the eutrophication of the lake. Eutrophication would influence the microbial community composition by increasing the concentration of compounds that are rich in phosphorus and nitrogen at a depth of $26 \mathrm{~m}$ in the MR region (Table 1).

Proteobacteria are involved in many biochemical processes that are vital to the environment. Proteobacteria perform the fixation of $\mathrm{CO}_{2}$ derived from organic and inorganic substrates via the Calvin-Benson-Bassham cycle; therefore, at lake Tucuruí, Proteobacteria, Cyanobacteria and methanogenic Archaea are responsible for maintaining the biochemical cycle of derived carbon from the organic biomass that was formed when the forest was submerged in the reservoir. Other communities that are important for this process could be best described as being located in the sediment and aphotic layers of the lake.

Alphaproteobacteria are characterized as being a metabolically flexible group and are present in environments with substantial variations in the $\mathrm{CO}_{2}$ and $\mathrm{O}_{2}$ concentrations. Betaproteobacteria are generally less flexible with respect to their metabolic strategies (Badger, 2008), and their primary habitat is freshwater that is normally still, photic and enriched with organic nutrients and compounds (Imhoff, 2006), which describes the environment under study and explains the predominance of this class at the reservoir.

In the gammaproteobacteria class, two of the four contigs were most closely related to Acinetobacter $(96 \%$ and $99 \%$ similarity). This genus includes gram-negative, non-fermentative bacteria that are found in soil, water, sewage, food and clinical settings as a nosocomial infectious pathogen (Towner, 2006). It should be noted, however, that there is a significant difference between the populations of this genus that are found in the clinical and environmental settings (Vallenet et al., 2008). Finally, members of the deltaproteobacteria class, which is the least abundant class in the reservoir, were classified as uncultivated environmental samples and are closely related to members of the same class found in 
metagenomes from acid lakes and Arctic sediments (Table 2).

\subsection{Archaea}

In the dendrogram, the Tucuruí archaeal reads form two clades that are related to the phyla Euryarchaeota and Crenarchaeota. It is important to detect and identify archaeal sequences in all types of environments to understand the ecological role of these microorganisms, which have long been considered to exist exclusively in extreme environments (Bintrim, 1997). The analysis of 16S rRNA genes have shown that Archaea are distributed in various environments, such as freshwater lakes, soil and marine environments (Schleper, 2005). According to Woese (1990), the phylum Euryarchaeota is represented by the orders Thermococcales, Methanococcales, Methanobacteriales and Methanomicrobiales, as well as the extreme halophiles. In the present study, only seven sequences were identified as belonging to the Euryarchaeota and correspond to the methanogenic archaeal group. The individuals represented by these seven sequences are involved in various biogeochemical cycles, especially the carbon cycle, because they perform methanogenesis, which is the production of methane from carbon dioxide, hydrogen, acetate, formate and other $\mathrm{C}_{1}$ compounds such as methanol, methylthiols and methylamines (Thauer, 1998). Approximately 133 of the sequences were similar to uncultivated archaea derived from studies involving a shotgun metagenomic approach (Seshadri, 2007), suggesting that despite the tremendous increase in the amount of sequences available in the database, the Archaea domain remains poorly characterized from a genomic perspective when compared to the Bacterial domain. OTU MFAP64 (Figure 03), which is represented by the sequence with accession number EU592947, is phylogenetically similar to an archaeal sequence from an environmental sample from Lake Gatun, Panama. This lake has similar characteristics as the HPS-Tucuruí reservoir, such as the temperature, salinity and depth of the sampling point. Lake Gatun and the HPS-Tucuruí reservoir share another important feature: both bodies of water were artificially created by flooding caused by the building of dams.

\section{Conclusions}

Amazonia is widely known to have one of the greatest megadiversities of fauna and flora in the world. In addition, the Tucuruí metagenome demonstrates that this biome also has a rich diversity of microbes, many of which are uncultivated. In the present study, we used culture-independent techniques and various bioinformatic tools to demonstrate that it was possible to obtain a profile of the prokaryotic diversity for the two sampling locations of the lake formed by the HEP-Tucuruí. The present study provides knowledge and understanding of the microdiversity that exists in landscapes created by the implementation of mega-hydropower enterprises in the Brazilian Amazon.

\section{Acknowledgement}

This work is part of the Program for Research and Development of the Electric Power Sector that is regulated by ANEEL and Centrais Elétricas do Norte do Brasil. The authors thank the National Council for Scientific and Technological Development (Conselho Nacional de Desenvolvimento Científico e Tecnológico - CNPq), the Federal University of Pará 
(Universidade Federal do Pará - UFPA) and the Higher Council for Scientific Research (Consejo Superior de Investigaciones Cientificas - CSIC).

\section{References}

Ashelford, K. E., Chuzhanova, N. A., Fry, J. C., Jones, A. J., \& Weightman, A. J. (2006). New Screening Software Shows that Most Recent Large 16S rRNA Gene Clone Libraries Contain Chimeras. Appl. Environ. Microbiol., 2 72, 5734-5741. http://dx.doi.org/10.1128/AEM.00556-06

Badger, M. R., \& Bek, E. J. (2008). Multiple Rubisco forms in proteobacteria: Their functional significance in relation to $\mathrm{CO}_{2}$ acquisition by the CBB cycle. J. Exp. Bot., 59, 1525-1541. http://dx.doi.org/10.1093/jxb/erm297

Benner, R., Opsahl, S., Chin-Leo, G., Richey, J., \& Forsberg, B. (1995). Bacterial carbon metabolism in the Amazon River system. Limnol. Oceanogr., 40, 1262-1270. http://dx.doi.org/10.4319/1o.1995.40.7.1262

Bintrim, S. B., Donohue, T. J., Handelsman, J., Roberts, G. P., \& Goodman, R. M. (1997). Molecular phylogeny of Archaea from soil. PNAS, 94, 277-282. http://dx.doi.org/10.1073/pnas.94.1.277

Borneman, J., \& Triplett, E. (1997). Molecular microbial diversity in soils from eastern Amazonia: evidence for unusual microorganisms and microbial population shifts associated with deforestation. Appl. Environ. Microbiol., 63, 2647-2653.

Chao, A., \& Yang, M. C. K. (1993). Stopping rules and estimation for recapture debugging with unequal failure rates. Biometrika, 80, 193-201. http://dx.doi.org/10.1093/biomet/80.1.193

Cole, J. R., Chai, B., Farris, R. J., et al. (2007). The ribosomal database project (RDP-II): introducing myRDP space and quality controlled public data. Nucl. Acids Res., 35, 169-172. http://dx.doi.org/10.1093/nar/gk1889

Crosbie, N. D., Pockl, M., \& Weisse, T. (2003). Dispersal and Phylogenetic Diversity of Nonmarine Picocyanobacteria, Inferred from 16S rRNA Gene and cpcBA-Intergenic Spacer Sequence Analyses. Appl. Environ. Microbiol., 69, 5716-5721. http://dx.doi.org/10.1128/AEM.69.9.5716-5721.2003

Curtis, T. P., Sloan, W. T., \& Scannell, J. W. (2002). Estimating prokaryotic diversity and its limits. PNAS, 99, 10494-10499. http://dx.doi.org/10.1073/pnas.142680199

Dedysh, S. N., Pankratov, T. A., Belova, S. E., Kulichevskaya, I. S., \& Liesack, W. (2006). Phylogenetic Analysis and In Situ Identification of Bacteria Community Composition in an Acidic Sphagnum Peat Bog. Appl. Environ. Microbiol., 72, 2110-2117. http://dx.doi.org/10.1128/AEM.72.3.2110-2117.2006

Ernst, A., Becker, S., Wollenzien, U. I. A., \& Postius, C. (2003). Ecosystem-dependent adaptive radiations of picocyanobacteria inferred from $16 \mathrm{~S}$ rRNA and ITS-1 sequence 
analysis. Microbiology, 149, 217-228. http://dx.doi.org/10.1099/mic.0.25475-0

Fearnside, P. M. (1989). Processos predatórios na floresta tropical úmida da Amazônia Brasileira [Predatory processes in the tropical rainforest of the Brazilian Amazon]. Estudos Avançados, 3, 21-35. http://dx.doi.org/10.1590/S0103-40141989000100003

Fearnside, P. M. (1993). Migration, Colonization and Environment: The Potential of Amazonian Ecosystems. Cad. Saúde Públ., $\quad 9, \quad 448-457$. http://dx.doi.org/10.1590/S0102-311X1993000400005

Fearnside, P. M. (1995). Hydroelectric Dams in the Brazilian Amazon as Sources of $\begin{array}{llll}\text { Greenhouse } \quad \text { Gases. } & \text { Environmental } & \text { Conservation, } & \text { 22, }\end{array}$ http://dx.doi.org/10.1017/S0376892900034020

Fearnside, P. M. (1997). Greenhouse-gas emissions from Amazonian hydroelectric reservoirs: the example of Brazil's Tucuruí Dam as compared to fossil fuel alternatives. Environmental Conservation, 24, 64-75. http://dx.doi.org/10.1017/S0376892997000118

Fierer, N., \& Jackson, R. B. (2006). The diversity and biogeography of soil bacterial communities. PNAS, 103, 626-631. http://dx.doi.org/10.1073/pnas.0507535103

Finlay, B. J. (2002). Global Dispersal of Free-Living Microbial Eukaryote Species. Science, 296, 1061-1063. http://dx.doi.org/10.1126/science.1070710

Fiore, M. F., Neilan, B. A., Copp, J. N., Rodrigues, J. L. M., Tsai, S. M., Lee, H., et al. (2005). Characterization of nitrogen-fixing cyanobacteria in the Brazilian Amazon floodplain. Water Research, 39, 5017-5026. http://dx.doi.org/10.1016/j.watres.2005.10.002

Gans, J., Wolinsky, M., \& Dunbar, J. (2005). Computational Improvements Reveal Great Bacterial Diversity and High Metal Toxicity in Soil. Science, 309, 1387-1390. http://dx.doi.org/10.1126/science. 1112665

Gonzalez-Toril, E., Llobet-Brossa, E., Casamayor, E. O., Amann, R., \& Amils, R. (2003). Microbial Ecology of an Extreme Acidic Environment, the Tinto River. Appl. Environ. Microbiol., 69, 4853-4865. http://dx.doi.org/10.1128/AEM.69.8.4853-4865.2003

Good, I. J. (1953). The Population Frequencies of Species and the estimation of population parameters. Biometrika, 40, 237-264.

Handelsman, J., Rondon, M. R., Brady, S. F., Clardy, J., \& Goodman, R. M. (1998). Molecular biological access to the chemistry of unknown soil microbes: a new frontier for natural products. Chemistry \& Biology, 5, R245-R249. http://dx.doi.org/10.1016/S1074-5521(98)90108-9

Hungria, M., Astolfi-Filho, S., Chueire, L., Nicolas, M. F., Santos, E. B. P., Bulbol, M. R., et al. (2005). Genetic characterization of Chromobacterium isolates from black water environments in the Brazilian Amazon. Letters in Applied Microbiology, 41, 17-23.

Imhoff, J. F. (2006). The Phototrophic Alpha-Proteobacteria. In Dworkin M, Falkow S, Rosenberg E, Schleifer K-H, Stackebrandt E (Eds.), The Prokaryotes (vol. 5, pp. 41-64). 
Springer. http://dx.doi.org/10.1007/0-387-30745-1_2

Junk, W. J., \& Mello, J. A. S. N. (1990). Impactos ecológicos das represas hidrelétricas na bacia amazônica brasileira [Ecological impacts of hydroelectric dams in the Brazilian $\begin{array}{llll}\text { Amazon basin]. Estudos } & \text { Avançados, }\end{array}$ http://dx.doi.org/10.1590/S0103-40142007000100009

Kemp, P. F., \& Aller, J. Y. (2004). Bacterial diversity in aquatic and other environments: what 16S rDNA libraries can tell us. FEMS Microbiol. Ecol., 47, 161-177. http://dx.doi.org/10.1016/S0168-6496(03)00257-5

Kersters, K. (2006). Introduction to the Proteobacteria. In Dworkin M, Falkow S, Rosenberg E, Schleifer K-H, Stackebrandt E (Eds.), The Prokaryotes (vol. 5, pp. 3-37). Springer. http://dx.doi.org/10.1007/0-387-30745-1_1

Lima-Bittencourt, C., Astolfi-Filho, S., Chartone-Souza, E., Santos, F., \& Nascimento, A. (2007). Analysis of Chromobacterium sp. natural isolates from different Brazilian ecosystems. BMC Microbiology, 7, 58. http://dx.doi.org/10.1186/1471-2180-7-58

Liolios, K., Mavromatis, K., Tavernarakis, N., \& Kyrpides, N. C. (2007). The Genomes On Line Database (GOLD) in 2007: status of genomic and metagenomic projects and their associated metadata. Nucl. Acids Res., 36, D475-D479. http://dx.doi.org/10.1093/nar/gkm884

Newton, R. J., Jones, S. E., Helmus, M. R., \& McMahon, K. D. (2007). Phylogenetic ecology of the freshwater Actinobacteria acI lineage. Appl. Environ. Microbiol., 73, 7169-7176. http://dx.doi.org/10.1128/AEM.00794-07

Nold, S. C., \& Zwart, G. (1998). Patterns and governing forces in aquatic microbial communities. Aquatic Ecology, 32, 17-35. http://dx.doi.org/10.1023/A:1009991918036

Percent, S. F., Frischer, M. E., Vescio, P. A., Duffy, E. B., Milano, V., McLellan, M., et al. (2008). Bacterial community structure of acid-impacted lakes: What controls diversity? Appl. Environ. Microbiol., 74, 1856-1868. http://dx.doi.org/10.1128/AEM.01719-07

Pruesse, E., Quast, C., Knittel, K., Fuchs, B. M., Ludwig, W., Peplies, J., et al. (2007). SILVA: a comprehensive online resource for quality checked and aligned ribosomal RNA sequence data compatible with ARB. Nuc. Acids Res., 35, 7188-7196. http://dx.doi.org/10.1093/nar/gkm864

Schleper, C., Jurgens, G., \& Jonuscheit, M. (2005). Genomic studies of uncultivated Archaea. Nat. Rev. Micro., 3, 479-488. http://dx.doi.org/10.1038/nrmicro1159

Schloss, P. D., \& Handelsman, J. (2005). Introducing DOTUR, a Computer Program for Defining Operational Taxonomic Units and Estimating Species Richness. Appl. Environ. Microbiol., 71, 1501-1506. http://dx.doi.org/10.1128/AEM.71.3.1501-1506.2005

Seshadri, R., Kravitz, S. A., Smarr, L., Gilna, P., \& Frazier, M. (2007). CAMERA: A Community Resource for Metagenomics. PLoS Biology, 5, e75. http://dx.doi.org/10.1371/journal.pbio.0050075 
Suzuki, M., Rappe, M. S., \& Giovannoni, S. J. (1998). Kinetic Bias in Estimates of Coastal Picoplankton Community Structure Obtained by Measurements of Small-Subunit rRNA Gene PCR Amplicon Length Heterogeneity. Appl. Environ. Microbiol., 64, 4522-4529.

Towner, K. (2006). The Genus Acinetobacter. In Dworkin M, Falkow S, Rosenberg E, Schleifer K-H, Stackebrandt E (Eds.), The Prokaryotes (vol. 6, pp. 746-758). Springer. http://dx.doi.org/10.1007/0-387-30746-X_25

Tringe, S. G., Zhang, T., Liu, X., Yu, Y., Lee, W. H., Yap, J., et al. (2008). The airborne metagenome in an indoor urban environment. PLoS ONE, 3, e1862.

Tundisi, J. G. (2007). Exploration of the hydroelectric potential of the Amazon. Estudos Avançados, 21, 109-117. http://dx.doi.org/10.1590/S0103-40142007000100009

Vallenet, D., Nordmann, P., Barbe, V., Poirel, L., Mangenot, S., Bataille, E., et al. (2008). Comparative Analysis of Acinetobacters: Three Genomes for Three Lifestyles. PLoS ONE, 3, e1805.

Venter, J. C., Remington, K., Heidelberg, J. F., Halpern, A. L., Rusch, D., Eisen, J. A. et al., (2004). Environmental Genome Shotgun Sequencing of the Sargasso Sea. Science, 304, 66-74. http://dx.doi.org/10.1126/science.1093857

Warnecke, F., Amann, R., \& Pernthaler, J. (2004). Actinobacterial 16S rRNA genes from freshwater habitats cluster in four distinct lineage. Environ. Microbiol., 6, 242-253. http://dx.doi.org/10.1111/j.1462-2920.2004.00561.x

Waterbury, J. B., Watson, S. W., Valois, F. W., \& Franks, D. G. (1986). Biological and ecological characterization of the marine unicellular cyanobacterium Synechococcus. In Platt T, Li WKW (Eds.), Photosynthetic Picoplankton. Dept. of Fisheries and Oceans, Ottawa. 214, $71-120$

Woese, C. R., Kandler, O., \& Wheelis, M. L. (1990). Towards a natural system of organisms: proposal for the domains Archaea, Bacteria, and Eucarya. PNAS, 87, 4576-4579. http://dx.doi.org/10.1073/pnas.87.12.4576

Wu, X., Xi, W., Ye, W., \& Yang, H. (2007). Bacterial Community composition of a shallow hypertrophic freshwater lake in China, revealed by $16 \mathrm{~S}$ rRNA gene sequences. FEMS Microbiol. Ecol., 61, 85-96. http://dx.doi.org/10.1111/j.1574-6941.2007.00326.x

\section{Copyright Disclaimer}

Copyright reserved by the author(s).

This article is an open-access article distributed under the terms and conditions of the Creative Commons Attribution license (http://creativecommons.org/licenses/by/3.0/). 